Gastroenterologia. 1954;82:I-IV

\title{
Contents, Vol. 82, 1954
}

\section{INDEX}

Balínt, J. A., and J. D. H. Slater, Deformity of the Pyloric Antrum. A Study in Practical Gastroenterology 330

Balzer, E., und K. Werner, Eine für die Zwecke der Pankreasfunktionsprüfung verbesserte Methode zur Bestimmung der proteolytischen Fermente im Duodenalinhalt 1

Bírnbaũn, D., The Effect of a New Parasympathetic Blocking Agent on Gastric Secretion and Gastrointestinal Motility in Man 225

Bois, P., vide H. Selye

Broicher, H.: Bemerkung zu der Arbeit «IO Jahre Erfahrungen mit Robuden» von O. Roth, erschienen in No. 5/6, Vol. 81 dieser Zeitschrift 175 Brummer, P., The Significance of Intestinal Decomposition Products of Cellulose on the Effect of Cellulose Laxatives 10

Calame, A., Laparoscopie, cholangiographie et photographie laparoscopiques 88

Fahrländer, H., und A. Schäffer, Messung der Erscheinungszeit von intravenös appliziertem Bromsulfalein in der Lebergalle als Funktionsprüfung des Choledochus. Bericht über 60 operativ kontrollierte Fälle 99

Friedrích, L., Erfahrungen bei 4500 eigenen Gastroskopiea, mit besonderer Rücksicht auf die Indikationen $\quad 300$

Haberlandt, W., vide K. Weíthaler

Hafter, E., Zur Diagnose der kleinen und reversiblen Hiatushemien. (IIhre

Abgrenzung von der Norm.) 76

Henníng, N., vide K. H. Kimbel

Jasinskí, B., vide W. Ott

Kapp, H., Zur zytologischen Gastritisdiagnose: Vergleich des Zellbildes des Magennüchterninhaltes mit dem histologischen und dem gastroskopischen Befund 117

Kimbel, K. H, H. Kínzlmeíer und N. Henning, Untersuchungen zur Magendurchblutung. I. Mitteilung: Versuche mit radioaktivem Phosphor 317 Kínzlmeíer, H., vide K. H. Kimbel

Lasch, F., Zur Behandlung von Leberparenchymkrankheiten mit Lebertotalextrakten 270

Lüthí, R., Magengeschwür und Hypophysenvorderlappen. Experimentelle Untersuchungen 162

Marion, P., Exploration radiologique et manométrique de Thypertension portale 65

Ott, W., und B. Jasinski, Nachuntersuchungen zum Thema Dumpingsyndrom und larvierter Eisenmangel 
Poli, M., Medical Aspects in Late Results of Partial Gastrectomy for Gastric and Duodenal Ulcers. Reichel-Polya and Finsterer-Hofmeister Modifica tions of Billroth II. (Review of 557 Cases.) 253

Porges, 0., Further Experience about Enteritis without Colitis

Part. 1. Description, Diagnosis and Therapy 129

Part. 2. Hypoglycemia; Enteritis and Biliary Symptoms 210

Rosenqvíst, H., and S.-G. Sjòberg, Gastro-Jejunocolic Fistula 285

Roth, 0., Erwiderung zu den Bemerkungen von Broicher 176

Sarasin, R., et B. Thommen, Etude de Гévacuation vésiculaire avec visuali sation du cholédoque 107

Schaeffer, A., vide H. Fahrländer

Selye, H., and P. Bois, Experimental Studies on the Influence of Corticoids and STH upon the Course of Obstructive Jaundice 193

Sjöberg, S.-G., vide II. Rosenquist

Slater, J. D. H., vide J. A. Balint

Steínítz, H., Gastritis hypertrophica gigantea 348

Thommen, B., vide R. Sarasin

Weithaler, K., und W. Haberlandt, Die kombinierte (internistisch-psycho-

therapeutische) Behandlung der Colitis ulcerosa 144

Werner, K., vide E. Balzer

Yanovsky, A., Les syndromes Ulcéro-Biliaires

Kasnistik - Case Report - Cagnlgtl $3 / 4 \pi$ e

Temesvári, A., Kongenitale Magenlues

233

Ciesellschafc $1 / 8$ berio hte - Society Transactions - Soci $^{3} / 8 t \phi s$

IVe Congrès de VAssociation des Socíêtês Nationales Europêennes et Médi-

terranéennes de Gastro-Entérologíe, 27 juin - 1 juillet 1954 à Paris

Socíété Beige de Gastro-Entérologíe (Seance extraordinaire du 30 mai 1954

à Gand) $\quad 46$

Schweizerische Gesellschaft für Gastroenterologie. Jahresversaminlung in

Brunnen am 23. Mai 1954. - Société Suisse de Gastro-Entêrologíe.

Seance du 23 mai 1954 à Brunnen 125

Compte-Rendu de la Session administrative de la Société Internationale de

Gastro-Entérologíe, tenue à Paris le 30 juin et le ler juillet $1954 \quad 180$

Kdiroi iai

Radiologie de Гintestin grêle

37

L'alcool est-il un aliment?

Groupes sanguins et maladies de $\Gamma$ estomac

Chirurgie abdominale preventive 306

$\mathrm{S}<$-ite der Ther«pie - Page de th $1 / 8$ rapentiqu $<1 / 8 \quad 49,181,239,308,365$

IteTei-ate $\cdot$ Abstracts $<$ Analyses

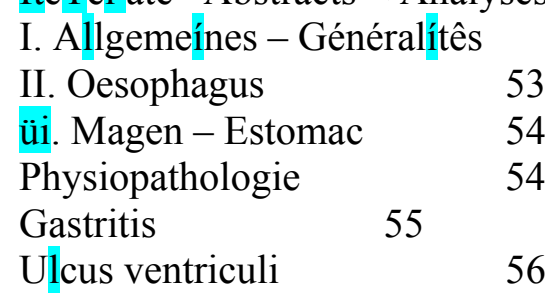

Gastritis 56 


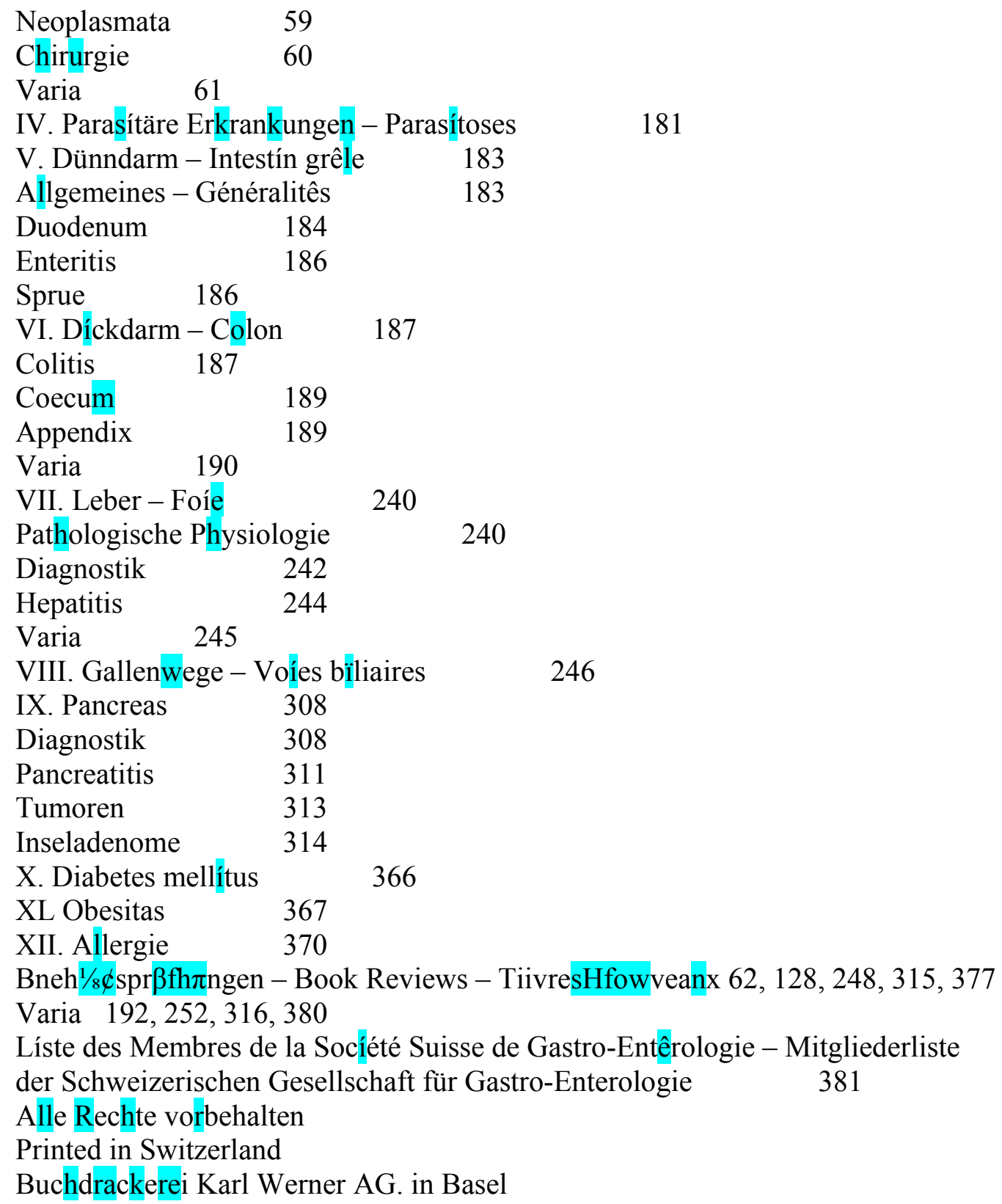

